\title{
MEMS AND Si MICROMACHINING FOR HIGH FREQUENCY APPLICATIONS
}

\author{
Linda P.B. Katehi \\ Purdue University \\ West Lafayette, IN 47907
}

\begin{abstract}
RF micromachining and MEMS technology promise to provide an innovative approach in the development of effective and low-cost circuits and systems, and is expected to have significant application in the development of low-cost antenna arrays and reconfigurable apertures. This paper presents a brief history and the state of the art in the development of RF MEMS devices, with primary emphases on switches, and of Si micromachined circuit components for use in high-performance, high-density, on-wafer packaged circuits.
\end{abstract}

\section{SUMMARY}

\section{a) RF MEMS Switches}

RF Micro Electrical Mechanical Structures (MEMS) has been identified as a technology, that has the potential to provide a major impact on existing RF architectures in sensors(radar) and communications by reducing weight, cost and size, and power dissipation. The impact of this technology to communication system cost, size and volume is expected to be a few orders of magnitude. Key MEMS devices for current RF architectures are switches in radar systems and filters in communications systems. From a system's standpoint, RF MEMS is enabling new RF System architectures. Future communications require increasing functionality and performance endurance requirements that can be addressed by the successful insertion of new highly integrated MEMS sensors and instruments.

In the last ten years, MEMS as applied to microwave and millimeter wave circuits have experienced an exponential growth. In 1991, Larson et al. described rotary MEMS switches with good performance at RF frequencies $[1,2]$. C.T. Nguyen demonstrated the successful development of MEMS HF filters in 1993-1994 [3] and Yao et al. demonstrated a surface micromachined series switch for telecommunications applications in 1995 [4]. Recently, shunt microwave switches have been developed in the $\mathrm{X}$ to $\mathrm{K} / \mathrm{Ka}$ band [5], [6] and [8]. These switches are usually electrostatic in nature and commonly driven by bias voltages in the 30-80 V range. Most recently, low actuation voltage switches requiring 9 Volts of DC actuation have been demonstrated [7], [9], [10] and have opened new directions in system implementation and development of communication systems architectures. These MEMS devices are primarily designed for low-loss applications that do not require fast rates such as airborne and/or deep space communication. The advantage of MEMS switches over their solid state counterparts such as FETs or PIN diodes is their extremely low series resistance and low drive power requirements.

For MEMS switches to become appropriate for wireless hand-held communications systems, actuation voltages less than 6 Volts are required. The primary goal of this section is to demonstrate the design, fabrication and DC as well as RF characterization of low- actuation-voltage electrostatic shunt microwave switches. Ultra low-loss, low actuation voltage RF MEMS switches have been successfully fabricated at the University of Michigan as shown in Figure 4. These switches are designed for use with finite ground coplanar waveguide, but the design methodology and fabrication approach are very general and can be utilized towards the development of any low-actuation voltage switch architectures. In order to lower the pull-in voltage of the structure, three different design goals may be pursued: (1) increasing the area of actuation, (2) diminishing the gap between the switch and bottom electrode, and (3) designing a structure with low spring constant. In the first case, the area can only be increased by so much before compactness becomes a prevailing issue. In the second case, the return loss associated with the RF signal restricts the gap. The third design goal is the one with the most flexibility, since the design of the springs does not considerably impact the size, weight, and/or RF performance of the circuit. Figure 1 shows a scanning electron micrograph (SEM) of a shunt switch design appropriate for operation with a Finite Ground CPW (FGCPW) interconnect. The development of RF MEMS devices continues at present in government laboratories, industry and academia with substantial emphasis on packaging and reliability.

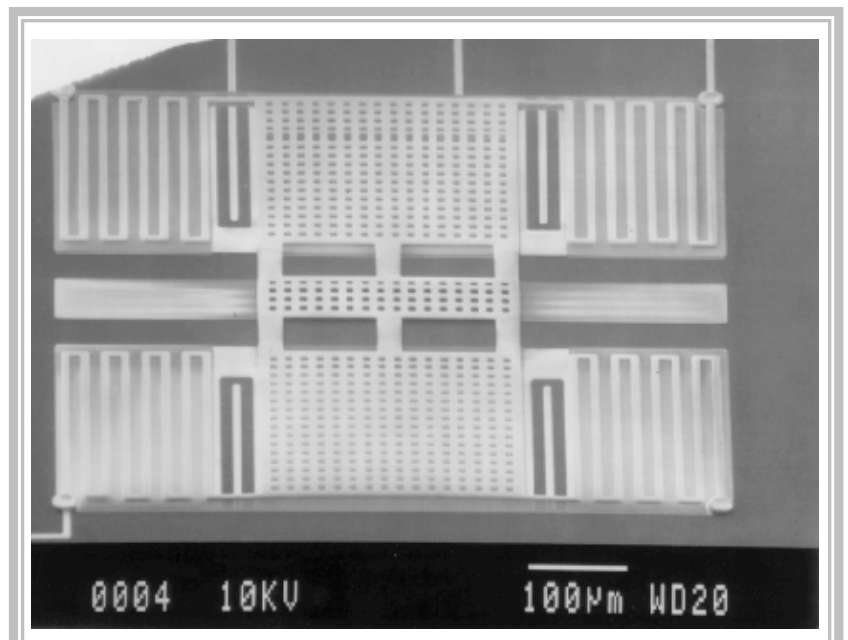

Figure 1: Low-Activation Voltage Switch Designed and Fabricated at the University of Michigan

\section{b) Three Dimensional Integration and Packaging Using Si Micromachining}

Emerging military, space, and commercial communications systems, as well as unique military applications in radar and missile seekers, are placing a high premium on low cost, small, light weight RF electronic components, while at the same time increasing demands for higher functionality. Systems are using higher and higher frequencies, in part for size considerations and in part for bandwidth. Commercial and military satellite, some commercial terrestrial, and space borne communications systems are operating in or being designed for the Ka-band $(25-40 \mathrm{GHz})$. 
On these satellite systems, electronic packaging can account for up to $30 \%$ of the overall spacecraft mass while the telecommunication subsystem can account for $15 \%$ or more of the dry mass. At the same time external organizational considerations are driving space agencies to reduce mission cost and failure probability (and hence reduce system complexity). Innovative approaches to the integration and packaging of RF systems are keys to achieving these goals.

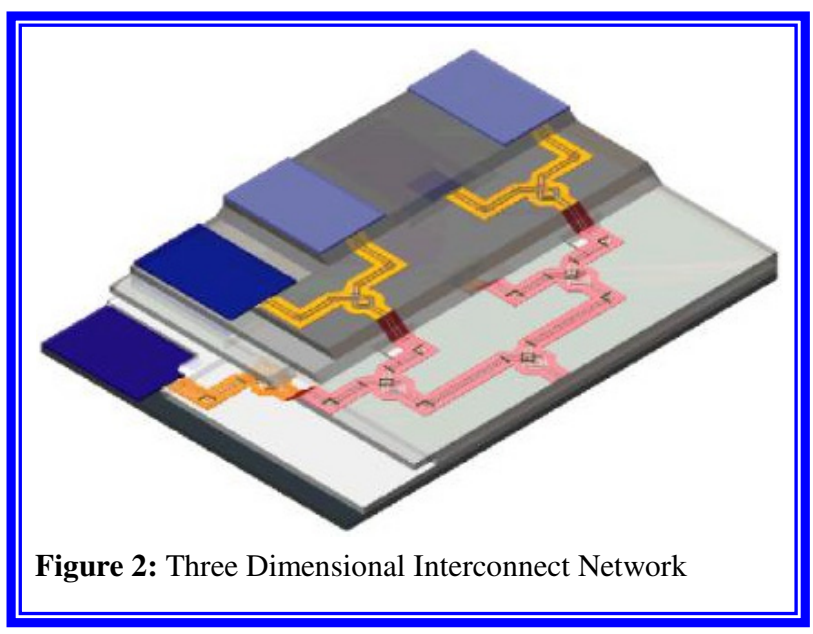

The framework of the micromachined integrated circuit is the collection of interconnects offered by this technology, each appropriate for specific applications. Micromachining techniques enable the fabrication of coplanar waveguide with the semiconductor material etched out between the conductor elements, thus substantially reducing dielectric loss and surface wave modes in the substrate. Layers of substrate can be added to the micromachined structure, with cavities cut to fit over the surface elements of the adjoining layer, providing shielding for the enclosed circuit elements. Metallization can be applied to the enclosure structure to further electromagnetically isolate the circuit elements. Isolations of $40 \mathrm{~dB}$ or better between transmission lines similar to those in Figure 2 and separated by $100 \mu \mathrm{m}$ have been demonstrated [11,12], providing an additional 20-30 dB greater than the isolation between conventionally fabricated lines, due to the micromachining of the substrate.

These shielded and micromachined circuit elements can then be geometrically laid out in a much denser circuit architecture. Using these approaches, radiation and substrate losses can be essentially eliminated, leaving only the ohmic loss of the metal. At higher frequencies, finite ground plane coplanar waveguide (FGC) and variations of it have been found to be most useful due to its ability to transition easily vertically through the wafers. However, depending on the applications, finite ground microstrip line could be the interconnect of choice.

\section{c) Lumped Passive Components}

At microwave and millimeter-wave frequencies (up to Ka band), a novel technology has been developed to design and realize high performance lumped passive components such as Wilkinson power dividers and hybrid couplers leading to substantial reduction in size. The basis of this novel technology is the development of high $\mathrm{Q}$, micromachined lumped components such as spiral inductors, metal-insulator-metal (MIM) capacitors and thin-film-resistors (TFRs). For example, by removing the Si wafer around a spiral inductor through selective etching as shown in Figure 7, inductors with a high resonant frequency ( $38 \mathrm{GHz}$ for a $1.5 \mathrm{nH}$ inductor), high Q-factor ( 30) and high linearity are possible. These micromachined spiral inductors can then be used to design dividers, hybrids and couplers at $\mathrm{X}-\mathrm{K}$ - and Ka-band with almost two orders of magnitude reduction in size, in addition to improved electrical performance in terms of insertion loss and bandwidth, with respect to standard printed circuit technology. Recent work has demonstrated, for the first time, X- and K-band power dividers with low loss $(0.6 \mathrm{~dB})$ and wide bandwidth $(25 \%$ bandwidth for 15 $\mathrm{dB}$ return loss) $[13,14]$. Due to the use of high-Q spiral inductors, the loss introduced by the lumped Wilkinson power divider is even lower than some distributed designs. One of the most important reasons for using lumped design is the smaller chip size.

Using lumped components to replace transmission lines, the chip size of the X-band design is reduced by a factor of six (to $0.3 \mathrm{~mm}$ $\mathrm{x} 0.5 \mathrm{~mm}$ ). These lumped components can be fabricated on a variety of III-V substrates and are expected to provide similar size reduction and performance improvement to a multistage MMIC module. Besides the narrow application to forming the basis for the RF power distribution network of the small sized MCMs, this component technology has the general potential to revolutionize high frequency RF circuit design and to lead to tremendous reductions in systems cost and size.

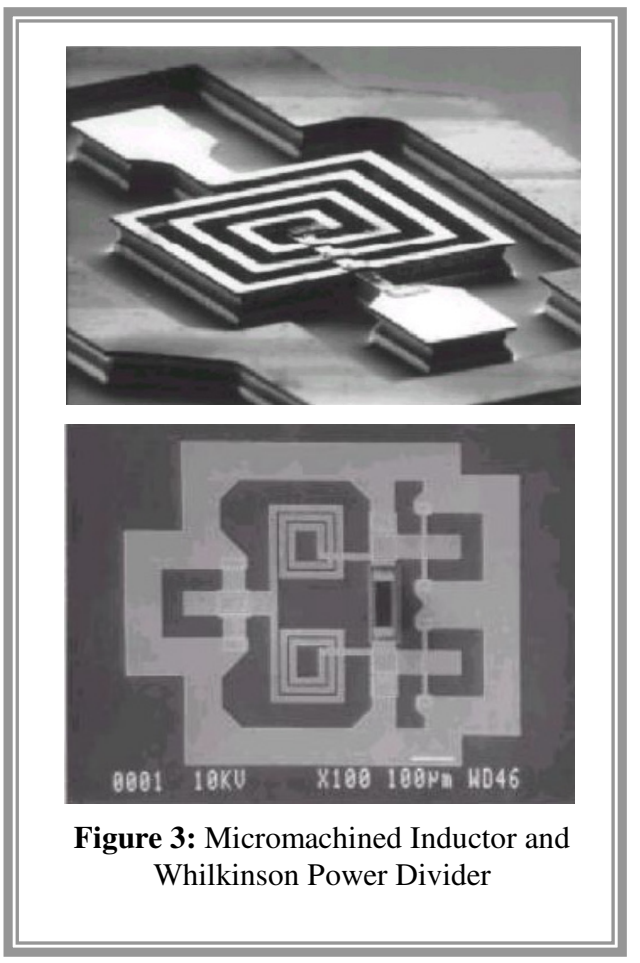

\section{d) Wafer-to-wafer Bonding}

The bonding of silicon wafers is a well-established commercial technology for applications such as power devices, silicon on insulator (SOI), MEMS sensors, die attachments, sealing, and other MEMS components [15]. Thermocompression bonding of gold-to-gold intermediate layers is the bonding method selected for the multi-layer architecture of Figure 4 because it can be achieved at a sufficiently low temperature that metalization and interconnects of the circuits on the various layers are undisturbed. This type of wafer-to-wafer bonding can be hermetic, so that the final multi-layer structure forms a complete hermetically sealed package. This wafer bonding technique is used for vacuum 
cavities in MEMS pressure sensors, and shows exceptional promise for RF circuits as well. This is the same technique used to form the RF bump electrical transitions described above, and these RF bump bonds form part of the mechanical bonding of the wafer layers as well as an electrical connection.

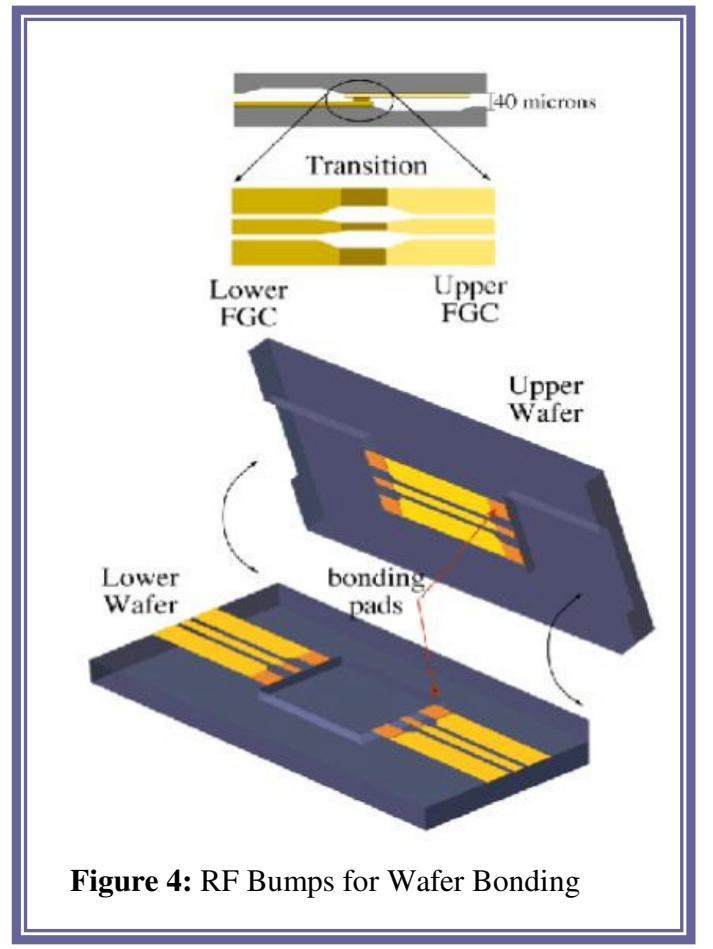

\section{CONCLUSIONS}

The micromechanical switch is without doubt the paradigm RF MEMS device. Within the last decade the RF community has experienced a growing plethora of MEMS switch designs. These designs have achieved a high level of RF performance, while maintaining ultra-low-power dissipation and large-scale integration. Due to the above characteristics, such RF MEMS devices should enable a wide variety of new system capabilities. With the ability to support any combination of electrical and micromechanical devices on a single chip, RF MEMS open an endless horizon of tantalizing possibilities for future designs and systems. In addition, the continual research on new design tools and methodologies, ensures that MEMS holds a tremendous promise in contributing heavily to the next generation of RF-based applications.

On the other hand, Si micromachining can provide on-wafer packaging and excellent performance. On-wafer $S$-parameter measurements of a $20 \mathrm{GHz}$ low noise amplifier with an integrated conformal package have been demonstrated. These measurements confirm that integration of a conformal package can be achieved in MMIC applications through the use of silicon micromachining techniques. The conformal package offers the benefit of RF shielding and isolation without detriment to the performance of the amplifier.

\section{ACKNOWLEDGEMENTS}

This work has been funded by DARPA MAFET II (under Raytheon) and by the Army Research Office.

\section{REFERENCES}

1. R. H. Hackett, L. E. Larson, and M. Melendes, "The Integration Of Micro-Machine Fabrication With Electronic Device Fabrication On III-V Semiconductor Materials," in Transducers Proceedings, pp.51-54, 1991.

2. C. T.-C. Nguyen, Micromechanical Signal Processors. PhD Thesis, University of California at Berkeley, 1994.

3. J. J. Yao and M. F. Chang, "A Surface Micromachined Miniature Switch For Telecommunications Applications With Signal Frequencies From Dc Up To 4 Ghz," in The 8th International Conference on Solid-State Sensors and Actuators Digest, pp. 384-387, 1995.

4. C. Goldsmith, T. H. Lin, B. Powers, W. R. Wu, and B. Norvell, Micromechanical Membrane Switches For Microwave Applications, IEEE MTT-S International Microwave Symposium Digest, pp. 91-94, 1995.

5. C. Goldsmith, J. Randall, S. Eshelman, and T. H. Lin, Characteristics Of Micromachined Switches At Microwave Frequencies, IEEE MTT-S International Microwave Symposium Digest, pp. 1141-1144, 1996.

6. Sergio Pacheco, Clark T. Nguyen, and Linda P. B. Katehi, "Micromechanical Electrostatic K-Band Switches," IEEE MTT-S International Microwave Symposium Digest, pp. 1569-1572, 1998. 7. C.L. Goldsmith, A. Malczewski, Z.J. Yao, S. Chen, J. Ehmke and D. H. Hinzel, RF MEMS Variable Capacitors for Tunable Filters, Special Issue on RF Applications of MEMS Technology, RF and Microwave Computer-Aided Engineering, v. 9, n. 4, pp. 362-374, July 1999.

8. Sergio P. Pacheco, Linda P. B. Katehi, and Clark T.-C. Nguyen, "Design of Low Actuation Voltage RF MEMS Switch," IEEE MTT-S International Microwave Symposium Digest, pp. 165-168, 2000.

9. Yongming Cai and Linda P.B. Katehi, "Wide Band Series Switch Fabricated Using Metal As Sacrificial Layer," European Microwave Conference, Paris, France, October 2000.

10. Jeremy B. Muldavin and Gabriel M. Rebeiz, "30 GHz Tuned MEMS Switches", IEEE MTT-S International Microwave Symposium Digest, pp. 1511-1514, 1999.

11. Katherine J. Herrick, Tom Schwarz and Linda P.B. Katehi, "Si-Micromachined Coplanar Waveguides for Use in High Frequency Circuits," IEEE Transactions on Microwave Theory and Techniques, Special Issue on Millimeter-Wave Technologies, Vol. 46, No. 6, June 1998, pp. 762-768.

12. Accuglass 512 Spin-on-Glass (SOG), Product Bulletin, Allied-Signal Inc., Planarization and diffusion products, $1090 \mathrm{~S}$. Milpitas Blvd., Milpitas, California 95035.

13. D. Kother, B. Hopf, Th. Sporkman and I. Wolf, "MMIC Wilkinson Couplers for Frequencies up to $110 \mathrm{GHz}$," 1995 IEEE MTT-S, pp. 663-666.

14. T. M. Weller, R.M. Henderson, K. J. Herrick L.P.B. Katehi, Three-Dimensional High Frequency Distribution Networks -Part I Optimization of CPW Discontinuities, accepted for publication in Transactions on Microwave Theory and Techniques, Oct. 2000.

15. Katherine J. Herrick and Linda P.B. Katehi, "RF W-Band Wafer-to-Wafer Transition," accepted for publication in IEEE Microwave Theory and Techniques, August 2000. 